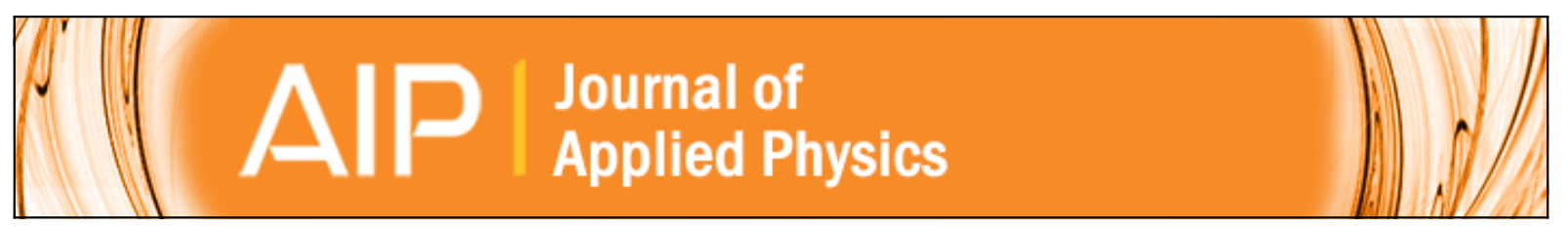

\title{
Phase transformation pathways in amorphous germanium under indentation pressure
}

S. Deshmukh, B. Haberl, S. Ruffell, P. Munroe, J. S. Williams, and J. E. Bradby

Citation: Journal of Applied Physics 115, 153502 (2014); doi: 10.1063/1.4871190

View online: http://dx.doi.org/10.1063/1.4871190

View Table of Contents: http://scitation.aip.org/content/aip/journal/jap/115/15?ver=pdfcov

Published by the AIP Publishing

\section{Articles you may be interested in}

Impurity-free seeded crystallization of amorphous silicon by nanoindentation

J. Appl. Phys. 110, 083707 (2011); 10.1063/1.3647587

Effect of hydrogen on nanoindentation-induced phase transformations in amorphous silicon

J. Appl. Phys. 106, 123511 (2009); 10.1063/1.3267853

Nanoindentation-induced phase transformation in relaxed and unrelaxed ion-implanted amorphous germanium J. Appl. Phys. 106, 093509 (2009); 10.1063/1.3255999

Phase transformations induced by spherical indentation in ion-implanted amorphous silicon

J. Appl. Phys. 100, 013520 (2006); 10.1063/1.2210767

Indentation-induced crystallization and phase transformation of amorphous germanium J. Appl. Phys. 96, 1464 (2004); 10.1063/1.1766414

\section{AlP $\left.\right|_{\text {Applied Physics }} ^{\text {Journal of }}$}

Journal of Applied Physics is pleased to announce André Anders as its new Editor-in-Chief 


\title{
Phase transformation pathways in amorphous germanium under indentation pressure
}

\author{
S. Deshmukh, ${ }^{1}$ B. Haberl, ${ }^{1}$ S. Ruffell, ${ }^{1}$ P. Munroe, ${ }^{2}$ J. S. Williams, ${ }^{1}$ and J. E. Bradby ${ }^{1}$ \\ ${ }^{1}$ Department of Electronic Materials Engineering, Research School of Physics and Engineering, \\ Australian National University, Canberra, Australian Capital Territory 0200, Australia \\ ${ }^{2}$ Materials Science and Engineering, University of New South Wales, Sydney, New South Wales 2052, \\ Australia
}

(Received 24 October 2013; accepted 1 April 2014; published online 16 April 2014)

\begin{abstract}
Nanoindentation-induced phase transformations have been studied in amorphous Ge thin films. These films initially tend to deform via plastic flow of the amorphous phase under load but at a critical pressure a sudden phase transformation occurs. This transformation, to a soft metallic ( $\beta$-Sn-like)-Ge phase confined under the indenter, is signified by a "pop-in" event on loading. Following "pop-in," the indentation tests fall into two distinct types of behavior. In one case, the rate of deformation with increasing load after "pop-in" increases, and the observed end-phase following complete unloading is observed to be predominately diamond-cubic Ge. In the other case, the deformation rate (slope of the loading curve) remains the same as that before "pop-in," and the end phases following unloading are found to contain predominantly unstable r8 and more stable hexagonal Ge phases. The different transformation pathways for these two cases are shown to be related to the probability that the soft ( $\beta$-Sn-like)-Ge phase volume, which suddenly forms at the transformation pressure, is either unconstrained by the indenter tip (the first case) or totally constrained under the indenter tip (in the latter case). (c) 2014 AIP Publishing LLC. [http://dx.doi.org/10.1063/1.4871190]
\end{abstract}

\section{INTRODUCTION}

The deformation behavior of amorphous silicon (a-Si) and germanium $(\mathrm{a}-\mathrm{Ge})^{1,2}$ under extreme pressure has been of interest for many years, in part, to compare with pressureinduced phase transformation characteristics of their crystalline counterparts. However, the nature of pressure-induced phase transformations appears to differ significantly between the crystalline and amorphous forms of both materials under diamond anvil cell (DAC) pressurization (see Refs. $2-5$ for $\mathrm{Si}$ and Refs. 3, 6-8 for $\mathrm{Ge}$ ) and also under indentation loading (see Refs. 9-11 for Si and Refs. 12-14 for Ge).

For crystalline germanium under DAC loading, it is well known that diamond cubic germanium (dc-Ge) undergoes a phase transition to a metallic $\beta$-Sn structure at a pressure of $\sim 10 \mathrm{GPa}^{1,2}$ On unloading ( $\beta$-Sn)-Ge has been reported to undergo a number of different phase transformations depending on unloading conditions. The following phases have been observed either during or following unloading: bodycentred-cubic bc8-Ge, tetragonal st12-Ge, a sparse rhombohedral $\mathrm{r} 8$-Ge phase, as well as end phases of hexagonal diamond (hd-Ge) and dc-Ge (Refs. 6, 7, 12, 15), along with some evidence for an a-Ge end phase. ${ }^{8}$ Nelmes et al. ${ }^{6}$ explained these different phase observations in terms of unloading rate in a DAC: slow unloading was reported to result in predominantly the stable st12-Ge phase, whereas fast unloading led to bc8-Ge, which is metastable and anneals at room temperature to hd-Ge. When a-Ge is the starting material, DAC-like loading again results in a metallic ( $\beta$-Sn)-Ge transition at 8-10 GPa, but several authors also report a metallic high density form of a-Ge under pressure. $^{8,16-18}$ On unloading, bc8-Ge is again observed
(Refs. 1, 18, 19), along with the re-appearance of a low density a-Ge phase ${ }^{8,17,18}$ in some cases, but unlike the crystalline $\mathrm{Ge}$ case, there is only sparse reference to $\mathrm{st} 12-\mathrm{Ge}^{8}$ or hdGe. ${ }^{19}$ Coppari et al. ${ }^{8}$ have suggested that voids observed in the type of a-Ge used in their experiments may account for crystalline/amorphous differences observed in their experiments. This may suggest that the differences between the transformation behavior of a-Ge and dc-Ge reported in the literature may relate to morphological and impurity content within the form of a-Ge used for the various DAC experiments.

Indentation with sharp or spherical diamond tips can also be used to induce phase transformations in both dc-Ge and a$\mathrm{Ge}$, but again there are substantial differences in the literature as to the deformation mechanisms and possible phase transformation pathways that can occur. For dc-Ge, there is clear evidence, in some cases, of a phase transformation to a metallic Ge phase under pressure ${ }^{19,20}$ but a variety of phases have been reported on unloading such as st12-Ge, ${ }^{12}$ traces of $\mathrm{r} 8-\mathrm{Ge},{ }^{21}$ and bc8-Ge, ${ }^{22}$ as well as a-Ge and dc-Ge end phases. ${ }^{15,22}$ However, other studies ${ }^{13,23,24}$ have shown that dc-Ge is difficult to phase transform under indentation loading and that "slow" loading favors deformation by slip, via dislocation motion, and twinning rather than an induced phase transformation. In terms of an a-Ge starting material, Patriarche et al. ${ }^{14}$ clearly showed that a phase transformation can take place under indentation loading and interpreted the observed end phases on unloading from Raman and TEM data as likely consisting of a mixture of dc-Ge and st12-Ge. Furthermore, the indentation and electron microscopy data of Oliver et al. ${ }^{15}$ showed that it is easier to induce a phase transformation in a-Ge compared with dc-Ge as a result of the ease of deformation by slip and twinning in the latter case. They observed a 
fully crystalline transformed zone within an a-Ge matrix that they interpreted as predominantly dc-Ge. More recently, Johnson et al. ${ }^{25}$ showed that indentation of a-Ge could result in a residual zone consisting almost entirely of a metastable r8-Ge phase on pressure release, but that this phase was unstable at room temperature. A further paper by Williams et al. ${ }^{26}$ showed that this unstable r8-Ge phase fully anneals at room temperature and pressure to hd-Ge. These two recent papers help to resolve some of the differences in the literature, but not the clear observation of dc-Ge, or alternatively st12-Ge, as the stable end phase in some other indentation studies. The present paper further clarifies the complex transformation behavior of Ge under pressure by reporting on two entirely different transformation pathways for a-Ge under indentation, one leading to dc-Ge (or st12-Ge), the other to r8 and hd-Ge phases.

\section{EXPERIMENTAL DETAILS}

For starting material, a-Ge was chosen for indentation experiments since it eliminates the competing deformation mechanism of slip and twinning exhibited by dc-Ge under load. Layers of a-Ge on Czochralski-grown Ge (100) wafers (dc-Ge) were made by self-ion-implantation using the ANU 1.7 MV NEC tandem accelerator. Three thicknesses of surface amorphous layer were formed: $\sim 700 \mathrm{~nm}, \sim 1000 \mathrm{~nm}$, and $\sim 1800 \mathrm{~nm}$. To form these layers, $\mathrm{Ge}^{+}$ions were implanted into the dc-Ge at liquid nitrogen temperature with the wafer surface normal $7^{\circ}$ to the incident beam and at an energy of $800 \mathrm{keV}$ to a fluence of $3 \times 10^{15} \mathrm{~cm}^{-2}$ to form the $700 \mathrm{~nm}$ thick layer, $1.3 \mathrm{MeV}$ to a fluence of $1 \times 10^{15} \mathrm{~cm}^{-2}$ to form the $1000 \mathrm{~nm}$ film, and $3 \mathrm{MeV}$ to a $1 \times 10^{15} \mathrm{~cm}^{-2}$ fluence to form the $1800 \mathrm{~nm}$ sample. Measurement of layer thicknesses was performed by Rutherford Backscattering Spectrometry (RBS), with ions channeled axially along the $\langle 100\rangle$ direction in the underlying dc-Ge, and by cross-sectional transmission electron microscopy (XTEM) to confirm that the amorphous layers were voidless and continuous to the surface.

Indentation was performed at room-temperature on all three samples using an ultra-micro-indentation-system 2000 (UMIS-2000) with diamond spherical indenter tips of radius $\sim 4.3 \mu \mathrm{m}$ or $\sim 20 \mu \mathrm{m}$. For the $\sim 4.3 \mu \mathrm{m}$ tip, maximum loads of $100 \mathrm{mN}$ and $120 \mathrm{mN}$ were applied to achieve a transformation event during loading and to avoid cracking in the $700 \mathrm{~nm}$ and $1000 \mathrm{~nm}$ specimens. A maximum load of $700 \mathrm{mN}$ was applied on the $1800 \mathrm{~nm}$ film using the $\sim 20 \mu \mathrm{m}$ tip to achieve a similar result. Arrays of 50-100 indents were made at each load for each film thickness. In some cases (see later), a sharp Berkovich tip was also used to induce transformations in these films up to a maximum load of $10 \mathrm{mN}$.

Residual indents were characterized by Raman spectroscopy and XTEM. Raman spectra from selected indents were recorded using a Renishaw 2000 Raman system with a $632.8 \mathrm{~nm}$ laser. The laser power was kept low to avoid annealing effects. For TEM analysis, cross-sections of selected indents were made with an FEI xT Nova NanoLab 200 dual-beam focused ion beam (FIB) with the ex-situ "liftout" technique. Prior to making the cross-sections, the arrays of indents were examined in-situ with scanning electron microscopy (SEM) within the FIB apparatus to image selected indents and to select desired indents for cross-sectioning. Samples were then coated with gold to prevent initial ionbeam-induced damage during FIB cross-sectioning. Platinum films were then deposited in-situ in the FIB to further protect the indented surface. The FIB-prepared cross-sections were imaged using a Philips CM 300 TEM operating at $300 \mathrm{kV}$. TEM samples were prepared for each condition and it should be noted that the TEM results exhibited excellent repeatability.

\section{RESULTS}

Figure 1 shows representative load-unload curves for indentation tests made in the $\sim 700 \mathrm{~nm}$ film [Fig. 1(a)] and $\sim 1000 \mathrm{~nm}$ film [Fig. 1(b)], using a $\sim 4.3 \mu$ m radius spherical tip. Typical curves for all samples featured a pop-in event, if loaded to a sufficient level, consistent with some previous reports in both dc-Ge and a-Ge. ${ }^{15}$ However, following a popin event, the indentation tests in both films $(\sim 700 \mathrm{~nm}$ and $\sim 1000 \mathrm{~nm}$ ) are observed to fall into two discrete deformation pathways, as indicated by the different slope of the loading curves following pop-in. In this paper, these two pathways are hereafter referred to as "family a" and "family b" behavior. From various sets of 50 indents performed in these samples, there was no consistent set of indentation conditions that determined into which of the two groups the data would reside. Quite surprisingly, there were no cases where the behavior was intermediate between "family a" and "family b" cases: the load-unload curves always fell into one or the
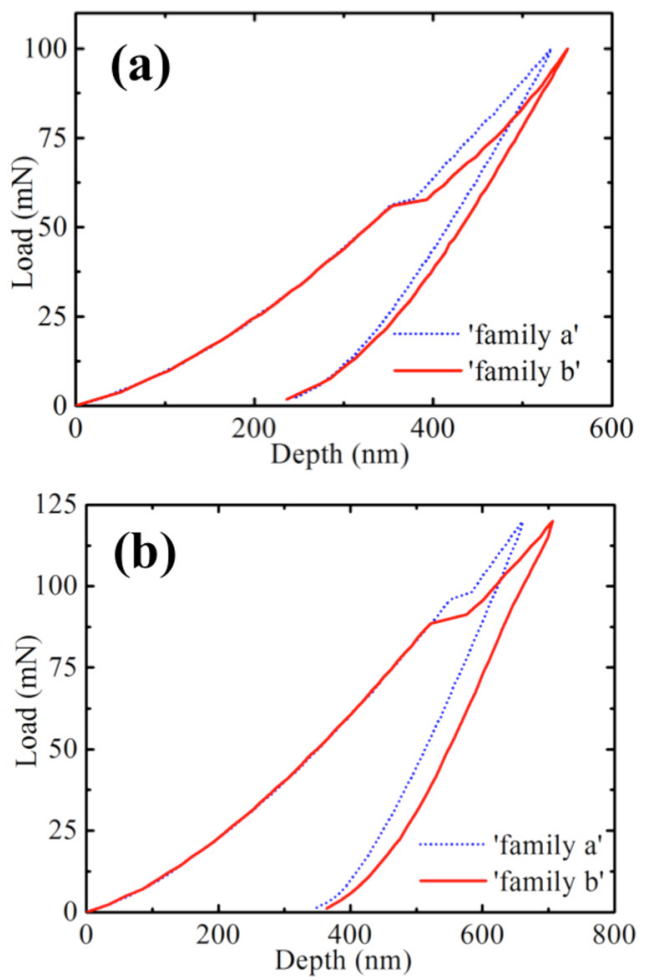

FIG. 1. Load-depth curves of indentations made in (a) $700 \mathrm{~nm}$ and (b) $1000 \mathrm{~nm}$-thick a-Ge films using a $\sim 4.3 \mu \mathrm{m}$ radius tip showing two deformation pathways, so-called "family a" and "family b." 
other behavior. For a $\sim 4.3 \mu \mathrm{m}$ radius spherical tip, the relative occurrence was roughly $60 \%-80 \%$ of indents exhibiting "family a" behavior.

Further details of the different "family a" and "family b" behavior includes the following. For both the $\sim 700 \mathrm{~nm}$ and $\sim 1000 \mathrm{~nm}$ films, "family a" indents are found to have a consistently smaller incremental depth at pop-in (and a smaller overall penetration depth at maximum load) compared to the "family b" indents. In addition, the load at which the pop-in event occurs for all tests in the same film, irrespective of "family a" and "family b" behavior, is remarkably consistent. This behavior suggests that pop-in, which we indicate below is the signature for a transformation to a $(\beta-\mathrm{Sn})-\mathrm{Ge}$ phase, is triggered identically irrespective of "family a" and "family b" behavior. Thus, the load at which the pop-in event occurs does not appear to be correlated with the deformation mode ("family a" or "family b"). Instead, the critical parameter in the loading curve that defines "family a" and "family b" behavior appears to be the magnitude of the pop-in event, with the penetration depth at pop-in consistently larger for the "family b" type curves. However, the pop-in load is film thickness dependent: for the $700 \mathrm{~nm}$ film it occurs at $56 \pm 2 \mathrm{mN}$, resulting in a depth at pop-in of $\sim 350 \mathrm{~nm}$, and in the $1000 \mathrm{~nm}$ film pop-in occurs at a load of $91 \pm 3 \mathrm{mN}$ and at a depth of $\sim 550 \mathrm{~nm}$. Thus, in both of these cases the pop-in occurs when the tip has penetrated $\sim 1 / 2$ of the film thickness, which we suggest later is related to the significant plastic deformation of the films during loading. In addition, the slope of the loading curve after pop-in for "family a" cases is consistently greater than that of the "family b" cases, suggesting different deformation processes after pop-in in the two cases.

Figure 2 shows a set of typical Raman spectra for the $\sim 700 \mathrm{~nm}$ film. A spectrum from an un-indented (background) area of this a-Ge film is also shown which exhibits a typical broad Raman band centered at $\sim 270 \mathrm{~cm}^{-1}$. For the "family a" Raman spectrum, there is evidence of extra Raman bands at $202 \mathrm{~cm}^{-1}, 225 \mathrm{~cm}^{-1}, 246 \mathrm{~cm}^{-1}$, and $280-295 \mathrm{~cm}^{-1}$. We have observed that some of these peaks

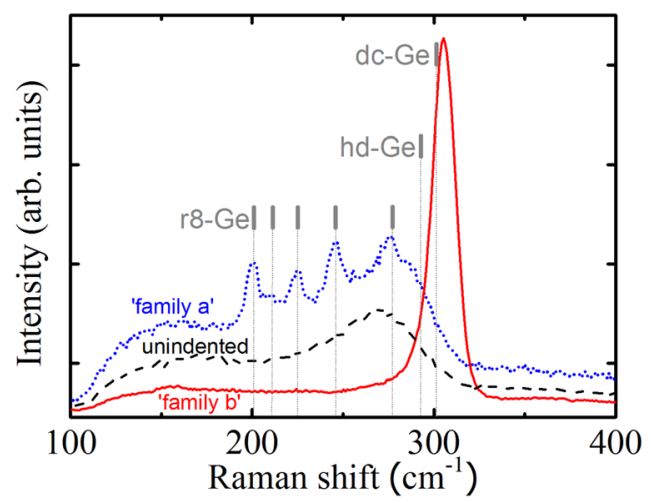

FIG. 2. Raman spectra taken from "family a" and "family b" indents loaded to $100 \mathrm{mN}$ using a $\sim 4.3 \mu \mathrm{m}$ radius tip in an $\sim 700 \mathrm{~nm}$ thick a-Ge film. A Raman spectrum from unindented a-Ge is shown for comparison. For optimal presentation the "family a" spectrum is multiplied by a factor of 5 and the unindented spectrum by 2.5 . The "family b" spectrum is unchanged. The previously reported ${ }^{25,26}$ experimental peak positions for $\mathrm{r} 8-\mathrm{Ge}$, hd-Ge, and dc-Ge are also indicated. are unstable, consistent with our very recent reports of unstable $\mathrm{r} 8-\mathrm{Ge}^{25}$ and its "annealing" to a more stable hd-Ge phase $^{26}$ at room temperature. Such bands have variously been attributed in the literature ${ }^{7,8,12}$ as arising from the st12, bc8, and $\mathrm{r} 8$ phases of $\mathrm{Ge}$, but we label them according to our recent assignments ${ }^{25,26}$ as $\mathrm{r} 8$ and hd-Ge phases in Fig. 2. The previously observed ${ }^{25}$ experimental position of the broad peak which characterizes the hd-Ge phase (straddling discrete peaks at $291 \mathrm{~cm}^{-1}, 305 \mathrm{~cm}^{-1}$, and $307 \mathrm{~cm}^{-1}$ ) has been labeled in Fig. 2. Finally, the Raman spectrum of "family b" indents is also shown in Fig. 2. This spectrum contains a single sharp peak close to $301 \mathrm{~cm}^{-1}$. We have confirmed this corresponds to the Raman signature of dc-Ge by comparing the "family b" spectrum in Fig. 2 with that for a pristine dc-Ge sample. We also note that this peak (slightly shifted from the expected position at $301 \mathrm{~cm}^{-1}$ presumably as a result of residual stress in this thin transformed a-Ge film) is readily distinguished from the broad hd-Ge band centered at a lower wave number as shown in Fig. 2.

XTEM samples were prepared from both "family a" and "family b" indents in the $\sim 700 \mathrm{~nm}$ and $\sim 1000 \mathrm{~nm}$ a-Ge films. Figure 3(a) shows a bright-field (BF) XTEM image of a "family a" indent in the $\sim 700 \mathrm{~nm}$ film indented to a load of $100 \mathrm{mN}$. The underlying dc-Ge substrate can be observed to deform via the generation of defects as previously described (slip and twinning). ${ }^{13}$ In the a-Ge layer, a clear region of phase-transformed material can be observed, extending through the entire thickness of the film. The inset to Fig. 3(a) shows a selected area diffraction pattern (SADP) from the transformed region. In this case, a selected area aperture was carefully positioned to be entirely contained in the indented region with no significant contribution from the dc-Ge substrate or the surrounding a-Ge. Indeed, this diffraction pattern is dominated by discrete reflections strongly indicating the presence of crystalline phases, along with a small amount of a-Ge. Indexing this pattern (see selected arrowed spots) indicates that all reflections correspond to hd-Ge lattice spacings, consistent with our recent report. ${ }^{26}$ Based on the instability of $\mathrm{r} 8$ and its transformation to hd-Ge at room
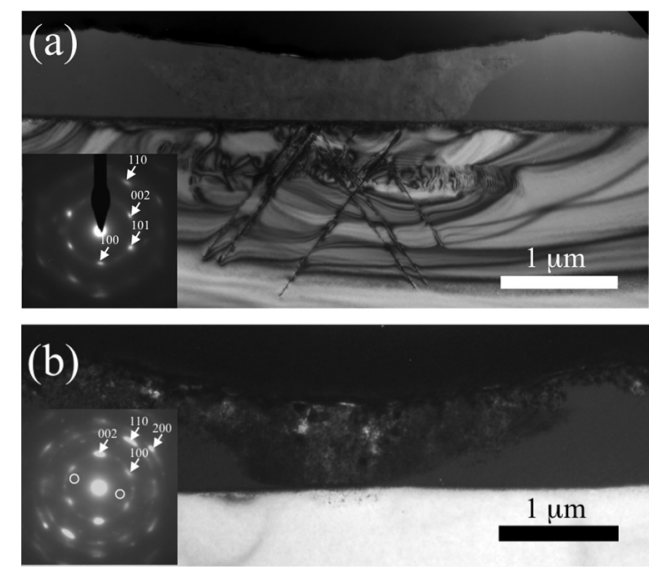

FIG. 3. Bright field XTEM images of "family a" indents in (a) an $\sim 700 \mathrm{~nm}$ film indented to $100 \mathrm{mN}$ and (b) an $\sim 1000 \mathrm{~nm}$ film indented to $125 \mathrm{mN}$. Insets show SADPs taken from the respective phase-transformed regions and indexed to hd-Ge. The weak circled spots in inset (b) have a d-spacing of $\sim 4.4 \AA$. 
temperature, it is not surprising that the XTEM data in Fig. 3(a), taken weeks after indentation, show almost entirely hd-Ge. Figure 3(b) shows a XTEM image and the corresponding SADP (inset) from a typical "family a" indent in the $\sim 1000 \mathrm{~nm}$ film. Again, hd-Ge is clearly observed in the SADP taken directly under the residual indent impression, with the other features essentially similar to the $700 \mathrm{~nm}$ film case. However, it can be noticed that there is only a slight amount of deformation in the underlying dc-Ge substrate in this thicker film case. In addition, there are some weak additional (circled) spots in the inset SADP of Fig. 3(b) which we discuss later.

XTEM images and corresponding SADPs for "family b" indents are shown for both the 700 and $1000 \mathrm{~nm}$ films in Figs. 4(a) and 4(b), respectively. They show clear crystallinity within the phase transformed volume as well as deformation in the underlying $\mathrm{dc}-\mathrm{Ge}$ in the case of the thinner $700 \mathrm{~nm}$ film. The most intense spots (not arrowed or labeled) in the corresponding SADPs [insets in Figs. 4(a) and 4(b)] can be indexed to predominately dc-Ge, as expected from the Raman data shown in Fig. 2. However, the SADPs also show evidence for some additional but weak diffraction spots [circled in Figs. 4(a) and 4(b)] that do not index to dcGe. The fact that there are no observable Raman signatures for these phases would suggest that there is only a trace amount of non-dc-Ge phases present in residual "family b" indents. We discuss the likely origin of these weak reflections later in this paper.

Figure 5 shows representative load/unload curves and associated Raman spectra for indentation of the thickest $(\sim 1800 \mathrm{~nm})$ a-Ge film. Here, indentation was performed using the $\sim 20 \mu$ m radius spherical tip principally to achieve a pop-in event, but to avoid cracking. For all indents, the slope of the loading curve after pop-in is consistent with "family a" behavior. Out of an array of 50 indents, only "family a" behavior was observed with the $\sim 20 \mu \mathrm{m}$ radius spherical tip. Figure 5(a) shows that, at a low maximum load of $450 \mathrm{mN}$, the load-unload curve is featureless, with no major pop-in event detected but considerable deformation
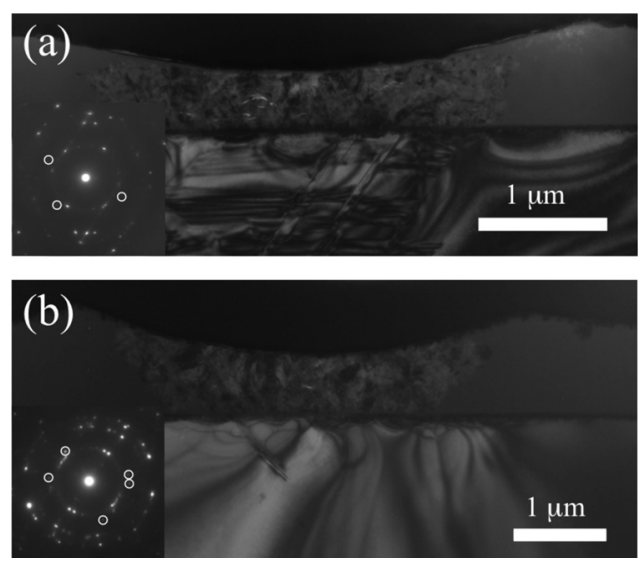

FIG. 4. Bright field TEM images of "family b" indents in (a) an $\sim 700 \mathrm{~nm}$ film indented to $100 \mathrm{mN}$ and (b) an $\sim 1000 \mathrm{~nm}$ film indented to $125 \mathrm{mN}$. Insets show SADPs taken from the respective phase-transformed regions which have been indexed predominately to dc-Ge. The weak circled spots indicate trace amounts of an additional phase.
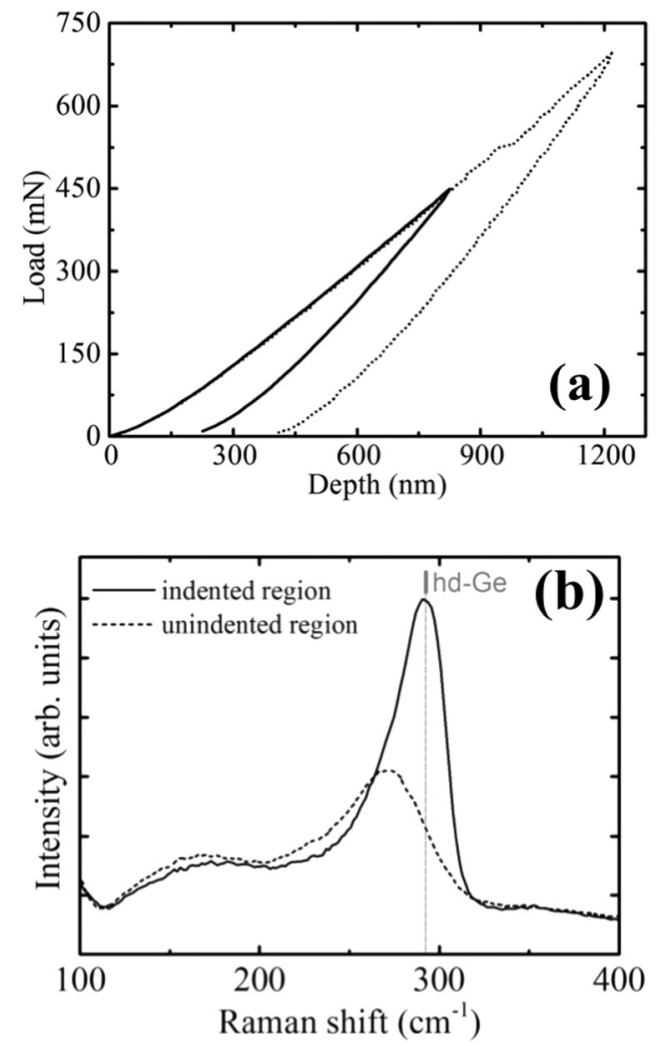

FIG. 5. (a) Load-unload curve from an $\sim 1800 \mathrm{~nm}$ thick a-Ge film indented with a $\sim 20 \mu$ m radius spherical tip to maximum loads of 450 and $700 \mathrm{mN}$. (b) Raman spectra from the $700 \mathrm{mN}$ indent with a spectrum from unindented a-Ge shown for comparison. The previously measured ${ }^{25}$ experimental Raman peak for hd-Ge is also indicated.

occurring as indicated by the residual penetration depth of $>200 \mathrm{~nm}$ following complete unloading. Although not shown, the Raman data from these low load indents indicate that no phase transformation has taken place under such indentation conditions. However, when the maximum load is increased to $700 \mathrm{mN}$, a pop-in occurs at $500-520 \mathrm{mN}$ load and at a penetration depth of $\sim 950 \mathrm{~nm}$ or at $\sim 1 / 2$ of the film thickness, similar to the thinner film cases. Raman data taken from indents produced at this higher load are shown in Fig. 5(b), where a major (broad) Raman peak centered at about $295 \mathrm{~cm}^{-1}$ can be observed. Based on our earlier "family a" behavior for the thinner films, we suggest that the dominant (stable) end phase is hd-Ge. Furthermore, we label our previously measured ${ }^{26}$ hd-Ge Raman peak position in Fig. 5 and note that it corresponds closely with the observed broad Raman band characterizing the stable end phase. Indeed, the fact that the $\mathrm{r} 8$ peaks are not clearly observed by Raman in the case of the thicker a-Ge film is almost certainly a result of the fact that the analysis was taken many days after indentation. This time lag between indentation and analysis would have led to transformation of any residual $\mathrm{r} 8$ to hd-Ge. ${ }^{26}$ Similar to the thin film cases, we find that $\mathrm{r} 8$ peaks can only be observed in the thick film case when Raman spectra are taken almost immediately after the indentation (not shown).

An XTEM image of an indent loaded to $700 \mathrm{mN}$ in the thick a-Ge film is shown in Fig. 6. Unlike the XTEM of the thinner films, a clear phase-transformed region is observed which does not extend throughout the entire a-Ge layer. 


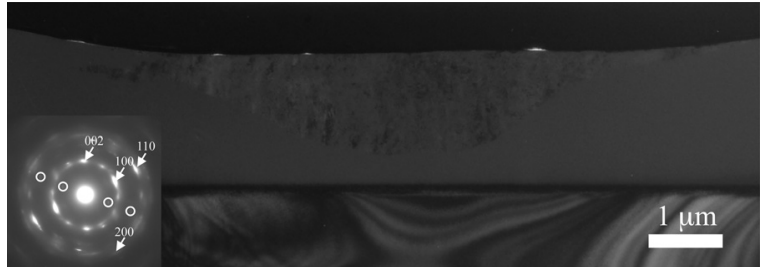

FIG. 6. Bright field TEM image of an indent in an $\sim 1800 \mathrm{~nm}$ thick a-Ge film made with a $\sim 20 \mu \mathrm{m}$ radius spherical tip to a maximum load $700 \mathrm{mN}$. Inset shows a SADP taken from the phase-transformed region where the most intense spots (selected spots arrowed) have been indexed predominately to hd-Ge. The weak circled spots indicate trace amounts of an additional phase that has a d-spacing of $4.5 \mathrm{~A}$.

There is no evidence of plastic deformation (i.e., twinning or dislocations) in the underlying substrate in this case. The SADP taken from the phase-transformed volume directly under the residual indent impression (see inset) shows predominant intense reflections with a d-spacing consistent with hd-Ge. However, as well as very weak a-Ge rings there are again some additional weak (circled) spots that indicate trace amounts of an additional phase, as we discuss later. Clearly, the XTEM results are consistent with the Raman data, indicating that the "stable" dominant end phase is hd-Ge.

It is also important to note that only one deformation pathway ("family a") is observed for the thick a-Ge film. This suggested to us that the large ( $\sim 20 \mu$ m radius) tip used for the indentation may have contributed to this behavior by confining a larger volume of transformed a-Ge between the tip and substrate. To establish whether such confinement of the transformed zone under a large spherical indenter may bias the behavior at pop-in to that of "family a," and, conversely, lack of confinement may result in "family b" behavior, we investigated the confinement argument more directly. We examined an array of indents in the SEM to determine if there was any evidence for "pile up" of extruded material in the "family b" cases. Typical SEM micrographs of "family a" and "family b" indents are shown in Fig. 7. It is apparent that the "family a" indent impression is smaller in area and does not exhibit any substantial pile up around the area of contact, suggesting that the phase transformed zone was effectively confined under the indenter. In contrast, the "family b" impression clearly shows extruded material beyond the contact area of the indenter. We note that high contrast in the SEM micrographs from "pile up" regions is not evidence for cracking since no cracks were observed in any of the XTEM micrographs. Indeed, all several hundred "family b" cases using spherical indenters that we examined always exhibited extensive pile-up behavior,
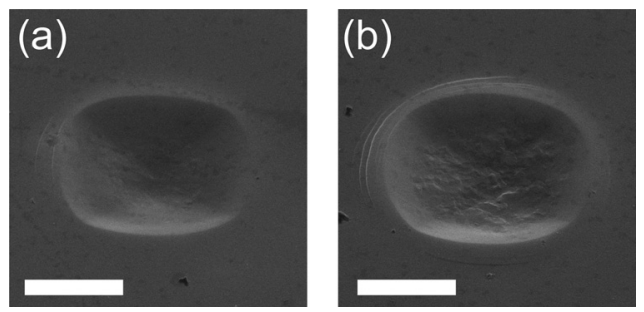

FIG. 7. Scanning electron microscopy images of indents from (a) "family a" and (b) "family b." Indented to maximum load of $100 \mathrm{mN}$ with a $4.3 \mu \mathrm{m}$ tip in an $\sim 700 \mathrm{~nm}$ thick a-Ge film taken at a $45^{\circ}$ tilt angle. Scale bars are $2 \mu \mathrm{m}$. strongly indicating that the transformed zone in these cases extended to the extremity of, or beyond, the contact area of the indenter at pop-in, consistent with possible extrusion of ( $\beta$-Sn)-Ge phase following pop-in. In contrast, all several hundred "family a" indents did not exhibit any appreciable pile up, consistent with constraint of the $(\beta-\mathrm{Sn})$-Ge phase under the indenter as was proposed above for the $20 \mu \mathrm{m}$ radius tip. Thus, our suggestion that "family a" behavior results from cases where the phase transformed zone is entirely confined under a spherical indenter, whereas "family b" behavior results from extrusion at pop-in, appears to be validated. Based on this behavior, we might expect that pointed Berkovich indenters are more likely to be biased towards "family b" behavior, which we have confirmed in a separate series of tests. All Berkovich impressions that we examined provided strong evidence for significant pile up (extrusion). We show a typical Berkovich impression to illustrate the strong extrusion in Fig. 8. An array of 50 indents were collected and in all cases they showed a pop-in and extensive extrusion consistent with "family b" behavior. We explain the consequences of this confined/unconfined behavior for subsequent phase evolution in the discussion section that follows.

\section{DISCUSSION}

Treating the loading behavior first, we note [as shown in the low maximum load vs penetration depth curve in Fig. 5(a)] that ion-implanted a-Ge appears to initially deform plastically prior to the pop-in load. However, the plastic deformation process is not sufficient to prevent continuous pressure build up during loading and a catastrophic phase transformation at higher indentation pressures ensues. This transformation process may be enhanced through densification of the a-Ge during indentation loading. Our data indicate that an a-Ge to $(\beta-\mathrm{Sn})$ Ge transformation occurs at pop-in which signifies that a significant volume of material transforms under the indenter to the denser and softer $(\beta-\mathrm{Sn})$-Ge phase. It is likely that the proximity of the underlying harder crystalline substrate contributes to accompanying densification of the a-Ge film and assists in reaching the necessary transformation pressure. In this regard, we note that the depth at pop-in increases with the thickness of the film from $350 \mathrm{~nm}$ for the $700 \mathrm{~nm}$ film, through $550 \mathrm{~nm}$ for the $1000 \mathrm{~nm}$ film and to $950 \mathrm{~nm}$ for the $1800 \mathrm{~nm}$ film. This

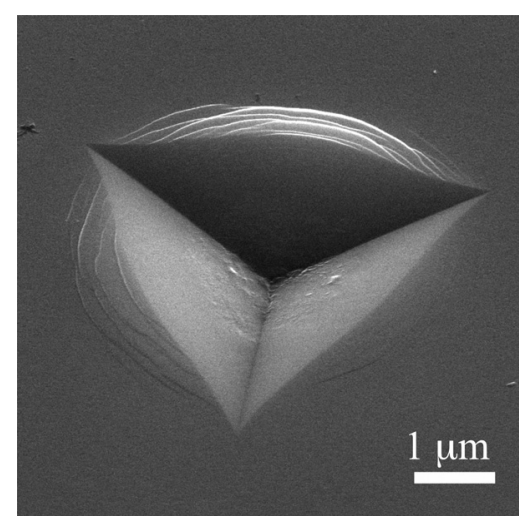

FIG. 8. Scanning electron microscopy image of a Berkovich indent loaded to $10 \mathrm{mN}$ in a $\sim 700 \mathrm{~nm}$ thick a-Ge film. The horizontal field width of the image is $6.2 \mu \mathrm{m}$ and the image was taken at a $45^{\circ}$ tilt angle. 
suggests that the thicker films can accommodate more substantial plastic deformation and can more effectively relieve the indentation stress. Hence, the proximity of the underlying dc-Ge substrate in the thinner film case reduces the degree of plastic deformation and phase transformation occurs at a lower load compared with thicker films. This behavior is somewhat similar to that in some a-Si films under indentation loading. In the $\mathrm{Si}$ case, it is found that ion implanted (unannealed) a-Si films deform plastically on indentation and it is difficult to initiate phase transformations in them. However, if the a-Si film is sufficiently thin and there is a harder underlying dc-Si substrate, a phase transformation can be induced at high loads. ${ }^{11}$ In such cases, the size of the depth increment at pop-in is of a similar scale to that of a-Ge in the current study and a large volume of a-Si material is observed to undergo transformation at pop-in, again somewhat similar to the a-Ge case. Furthermore, when a-Si films are annealed to allow structural relaxation to occur, little plastic deformation occurs and the films, regardless of thickness, undergo phase transformation at a similar load as those necessary for c-Si. Such structural relaxation is thought to result in a reduction in the dangling bond density, thus producing a form of a-Si that is close to a fully coordinated continuous random network. ${ }^{27}$ Thus, by comparison with the indentation behavior of a-Si films, we suggest that our asimplanted a-Ge films ${ }^{27}$ are partly relaxed and thus undergo some plastic deformation on indentation, but eventually undergo phase transformation at high loads significantly easier than does totally unrelaxed a-Si.

If pop-in signifies the transformation of a large volume of a-Ge under the indenter to a ( $\beta$-Sn-like)-Ge phase, why do we have two different transformation pathways, characterized by "family a" and "family b" behavior post pop-in? Having established that "family a" behavior is characterized by confinement of the transformed zone under the indenter and the "family b" case with extrusion of transformed material at pop-in, we first examine the post pop-in loading behavior to give further detail of the transformation processes. We note that the shape of the loading curves after popin is consistent with confinement and non-confinement of respective "family a" and "family b" cases. In particular, a smaller pop-in is observed for the confined "family a" case compared with the unconstrained "family b" case that exhibits extrusion at pop-in. Furthermore, after pop-in "family a" curves have a similar slope to that before "pop-in," consistent with continued deformation of surrounding a-Ge, whereas "family b" curves exhibit a smaller slope indicative of more rapid deformation (or extrusion of a softer $(\beta-\mathrm{Sn})$ Ge phase) with increasing load. We suggest that the main determinant of "family a" or "family b" behavior results from the statistical nature of a sudden phase transformation of a large volume of a-Ge at pop-in. There will be a finite probability that some of the metallic ( $\beta$-Sn)-Ge phase nucleates at or close to the indenter contact radius (hence extrusion of this unconstrained soft phase) and "family b" behavior will result. The probability for this to happen increases as the radius of the indenter tip decreases, as we find, with sharp pointed indenters strongly favoring "family b" behavior.

We are now ready to address the question: what (transformation) changes are occurring after pop-in in the "family a" and "family b" cases? Treating the "family b" case first, we again note the comparison with unrelaxed a-Si under indentation. For a-Si, the unconstrained $(\beta-\mathrm{Sn})-\mathrm{Si}$ phase that flows out from under the indenter transforms suddenly to a$\mathrm{Si}$, which is readily observed in the pile up regions surrounding the indents from XTEM data. ${ }^{10}$ There is some evidence that this may also happen for "family b" behavior in a-Ge, where the XTEM data (see Fig. 6) indicates not only dc-Ge, but also some a-Ge in the pile up region, although it is difficult to distinguish whether this material arises from outflow of the original amorphous film or is a result of the transformation of extruded ( $\beta$-Sn)-Ge. However, unlike the Si case, the transformed $(\beta$-Sn)-Ge that remains under the indenter clearly transforms predominantly to dc-Ge following pop-in during the loading cycle and/or during unloading. We suggest that the sudden pressure release in the unconstrained $(\beta$ $\mathrm{Sn}$ )-Ge component at pop-in causes some of it to transform directly to dc-Ge (possibly with some residual a-Ge). Next, this dc-Ge acts as a crystalline seed to favor direct nucleation of the lower free energy dc-Ge phase first within the partially confined region near the edge of the indenter tip and then for transformation of the remaining $(\beta-\mathrm{Sn})-\mathrm{Ge}$ under the indenter tip during the early stages of unloading.

It is worth noting at this point the analogy between our indentation observations of "family b" phase transformation behavior in this current study and the previously studied explosive crystallization phenomenon ${ }^{28,29}$ in a-Ge films under rapid mechanical action. The explosive crystallization process is thought to arise in thick a-Ge films, where the mechanical (strain) energy initiates crystallization to dc-Ge at room temperature, which in turn releases heat that then thermally drives the crystallization front into the a-Ge layer if the underlying thermally conducting substrate is sufficiently remote. We suggest that in the explosive crystallization process, a pressure-induced phase transformation to metallic $(\beta$ Sn)-Ge may be a possible intermediate step that "triggers" a transformation to dc-Ge when it becomes unstable under very sudden pressure release. In our indentation case, the very small volume of $(\beta-\mathrm{Sn})-\mathrm{Ge}$ that transforms to dc-Ge may be insufficient to generate enough heat (noting our relatively thin film cases) to sustain an explosive event into surrounding a-Ge. However, once the dc-Ge phase forms within the extruded material, we suggest that there is a driving force (related to the heat of crystallization ${ }^{28,29}$ ) for this phase to propagate upon pressure release in a continuous fashion (into the remaining constrained $(\beta-S n)-G e$ phase under the indenter) when the metallic phase becomes unstable. To confirm this proposal, it may be worth exploring in a subsequent study the synergies between the two processes (indentationinduced crystallization and explosive crystallization) in thick a-Ge films subject to ultra-fast, high load indentation conditions. Indeed, it may be possible that the initiation of explosive crystallization may be a factor that dictates the different deformation pathways, however, further in-situ techniques (such as electrical or x-ray measurements) would be needed to further investigate this possibility.

Turning now to the "family a" case, we suggest that the constrained $(\beta$-Sn)-Ge phase remains stable during loading but becomes unstable upon unloading similar to the DAC 
experiments referred to earlier. We note that the Raman data indicate that the dominant end phase is either $\mathrm{r} 8$ or hd-Ge (in keeping with the previously reported ${ }^{26}$ instability of the $r 8$ phase and its transformation at room pressure and temperature to hd-Ge). The fact that pop-out events are not observed at all during unloading may indicate that no sudden transformation occurs and there is a progressive transformation from $(\beta-\mathrm{Sn})-\mathrm{Ge}$ to the $\mathrm{r} 8$ phase on pressure release. As we suggested previously, ${ }^{25}$ the fact that there is no strong evidence for the bc8-Ge phase in our work may be a result of the residual stress within the indentation volume that preserves the r8 phase on full unloading. However, we observe that the r8Ge phase is unstable, and transforms predominantly to hd-Ge as shown by both the Raman data for the thick film case (Fig. 5) taken several days after indentation and the TEM data (see Figs. 3 and 6). It is not possible to ascertain unequivocally from data in this study whether bc8-Ge is an intermediate phase in this rapid transformation of $\mathrm{r} 8 \mathrm{-Ge}$ to hd-Ge, although the weak extra reflections observed in TEM SADPs may suggest that this is the case, as we discuss below.

It is appropriate now to discuss the possible origin of the weak additional spots observed in several of the SADPs that correspond to both "family a" and "family b" behavior. The diffuse nature and small number of these additional spots make them difficult to unequivocally identify but, when indexed, they appear to correspond to a lattice $\mathrm{d}$ spacing of around 4.4 to $4.5 \AA$. This suggests that they may correspond to either the st12 or bc 8 phases of Ge. However, on further analysis, we note that the number and symmetry of the weak reflections in the "family b" SADPs (see Fig. 4) suggest that they are more likely to correspond to the st12-Ge phase. In contrast, the extra spots in the "family a" cases, which are more diffuse and fewer in number, exhibit a symmetry more like that expected from the bc8-Ge phase (see Figs. 3(b) and 6). In relation to the possible presence of trace st12-Ge in "family b" indents, we note that previous indentation studies $^{12,14}$ that report the observation of st12-Ge have been in cases where extreme loading conditions and pointed indenters were used to induce a phase transformation, conditions that are highly likely to result in $(\beta$-Sn)-Ge extrusion from under the indenter tip at pop-in, as we discuss later. Hence, we leave open the possibility that some small volumes of $(\beta$ $\mathrm{Sn}$ )-Ge remaining under the indenter tip in our "family b" cases may also transform directly to st12-Ge in regions of high shear stress. In the case of "family a" indents we note that based on previous DAC studies, ${ }^{6}$ we might have expected to observe bc8-Ge since under hydrostatic unloading $\mathrm{r} 8 \mathrm{-Ge}$ is found to transform to the bc8 phase prior to reverting to the stable hd-Ge phase. Hence, it is reasonable to propose that the weak spots in the "family a" SADPs arise from trace amounts of the bc 8 phase.

Having reviewed the likely micro-structural transformations occurring during indentation based on our SEM, Raman, and TEM observations, we now summarize our proposed transformation pathways for "family a" and "family b" behavior in Fig. 9. Initially, plastic deformation occurs on loading but the local pressure under the indenter continues to build with increasing load until a catastrophic transformation of a large volume of a-Ge to metallic ( $\beta$-Sn)-Ge occurs under the indenter at pop-in when the tip penetration depth is around half the film thickness. Two separate types of behavior ensue. In the case of "family a," there is a smaller pop-in and the metallic phase is totally constrained under the indenter. On unloading, $(\beta$-Sn)-Ge progressively transforms to the $\mathrm{r} 8$ phase which is unstable and further transforms at room pressure and temperature to the more stable hd-Ge phase. We are not able to establish whether bc8-Ge is an intermediate phase in this rapid transformation, although there may be some evidence from weak additional spots in TEM SADPs that this is indeed the case. We have shown a dotted line in Fig. 9 for the r8 to hd-Ge transformation pathway to leave open the possibility of such an intermediate phase. In the case of "family b," a large pop-in signifies that the soft $(\beta-\mathrm{Sn})-\mathrm{Ge}$ phase is extruded from under the indenter and we suggest that this unconstrained material undergoes a

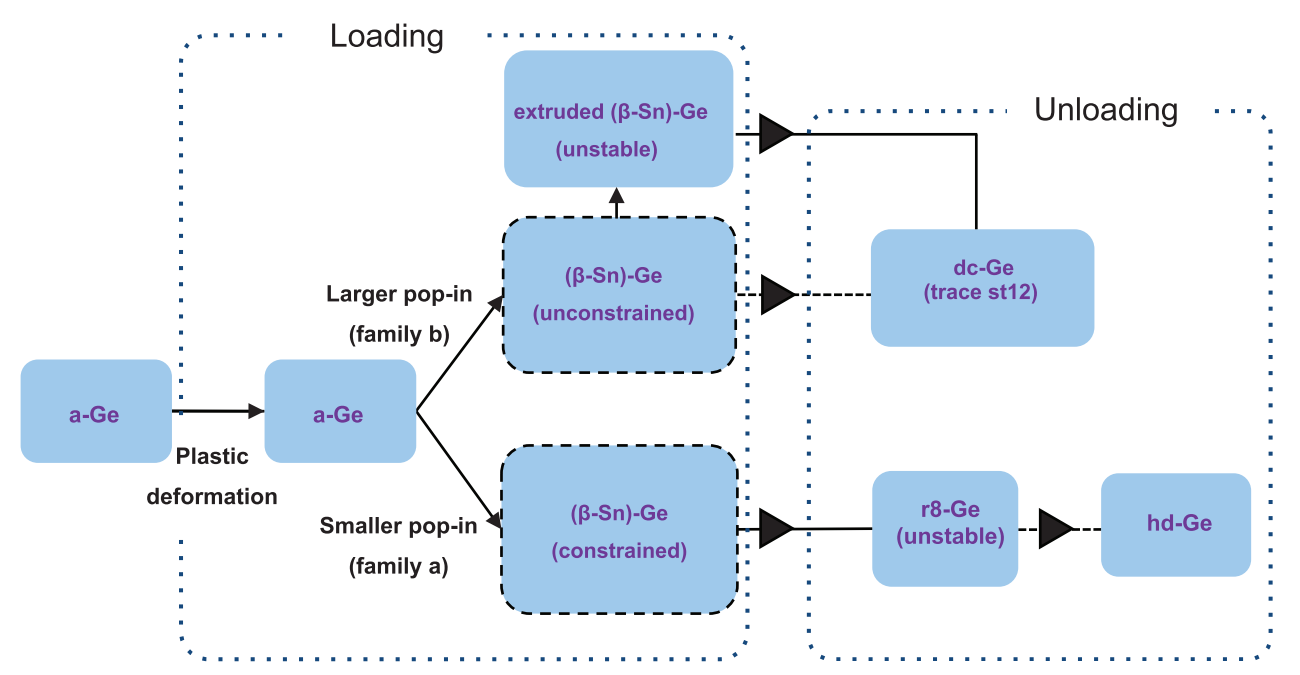

FIG. 9. Schematic showing the deformation pathways associated with the indentation of a-Ge found in the current study. The dashed transformation pathways indicate that we are unsure whether there are intermediate phases associated with the "family b" transformation from ( $\beta$-Sn)-Ge to dc-Ge on unloading and also for the "family a" transformation from r8-Ge to hd-Ge at room temperature. 
transformation directly to dc-Ge (with possibly some a-Ge) within the extruded material near the edge of the indenter contact area. We suggest that the sudden transformation to dc-Ge seeds nucleation of further dc-Ge, first within the partly constrained ( $\beta$-Sn)-Ge near the edge of the indenter contact, then upon progressive pressure release through the remaining $(\beta-\mathrm{Sn})$-Ge phase from the interface of $(\beta-\mathrm{Sn})-\mathrm{Ge}$ in contact with surrounding dc-Ge. We suggest that this process may be somewhat akin to the previously observed explosive crystallization phenomenon in a-Ge.

However, the "family b" behavior may be more complicated than Fig. 9 suggests and we have used a dotted line to denote the transformation from $(\beta-\mathrm{Sn})-\mathrm{Ge}$ to $\mathrm{dc}-\mathrm{Ge}$ on unloading to leave open other possibilities. For example, we are not able to determine whether there are any intermediate phases between $(\beta-\mathrm{Sn})-\mathrm{Ge}$ and the final dc-Ge phase. In this context, the observation of likely trace amounts of the st12Ge phase in residual "family b" indents warrants some comment. We first note that based on DAC results, Nelmes et $a l .{ }^{6}$ proposed that slow unloading from the $(\beta-\mathrm{Sn})-\mathrm{Ge}$ phase resulted in a dominant st12-Ge phase, whereas fast unloading favored transformation to bc8-Ge. This is not consistent with our current indentation data where confined and slower depressurization conditions of "family a" behavior show dominant r8 and finally hd-Ge phases. Rather, it is our rapid depressurization "family b" cases that appear to exhibit trace amounts of st12. Indeed, previous indentation studies of dc-Ge have also clearly identified the st12-Ge end phase under conditions of substantial extrusion and rapid depressurization. ${ }^{12,30}$ This disagreement between DAC understanding and indentation behavior poses the question as to whether the high shear stress conditions of indentation are the reason for the discrepancy. To help resolve this issue, we have recently undertaken further DAC studies of dc-Ge to clarify the influence of shear stress and unloading rate on the transformation from $(\beta-\mathrm{Sn})-\mathrm{Ge}$ under depressurization. ${ }^{31}$ Results clearly show that the use of a gas pressure medium that provides quasi-hydrostatic conditions produces the r8 phase on depressurization regardless of the rate, whereas with no pressure medium (i.e., large pressure gradients and high shear) st12 is observed to be the end phase. This result suggests that high pressure gradients and shear stress during unloading can favor st12-Ge, which is now consistent with the current study and the previously available indentation behavior.

Finally, it is appropriate, in light of our results in the current paper, to further comment on and clarify some of the apparent inconsistencies in previous indentation studies of Ge. We believe that such inconsistencies are a result of three issues: first, that the majority of previous studies used dc-Ge as the starting material, where extreme indentation conditions are needed to induce phase transformations; second, that wrong assignment of Raman peaks has occurred in some cases; and third, that there has been a lack of appreciation of separate transformation pathways, as shown in the current study. For dc-Ge as starting material, appropriate choice of indenter shape, maximum load and loading rate is crucial to obtaining a phase transformation rather than simply inducing deformation via plastic flow of the crystalline Ge through slip and twinning. Indeed, sharp indenters, high loads and/or fast loading rates were observed to favor deformation by phase transformation. ${ }^{20-24}$ When using sharp indenter tips, Jang et al $^{22,32}$ noted that extensive extrusion of material outside of the contact area always accompanied a phase transformation and they suggested that this observation in itself can be used to infer that a phase transformation had occurred. Using Raman mapping they showed that the extruded material $^{22}$ contained a-Ge, but there was also strong evidence for transformation of some of this phase to nanocrystalline dc-Ge. ${ }^{32}$ Gogotsi and co-workers ${ }^{12,21,23}$ and Oliver et al. $^{30}$ also observed a-Ge around the residual indent area (extruded material) when phase transformation of dc-Ge took place under indentation. However, since the starting material was dc-Ge in all of these studies, from Raman spectra it was extremely difficult to distinguish the presence of (nanocrystalline) dc-Ge in the end phase from the starting dc-Ge phase. Despite this limitation of detecting dc-Ge as an end phase, we suggest that these observations of extruded material are consistent with the "family b" behavior observed in the current study.

Another limitation of using dc-Ge as the starting material is that extreme indentation conditions are needed to force a phase transformation to occur in light of the preference for the material to plastically deform via slip/twinning. As indicated above, sharp indenter tips and fast loading rate conditions will favor extrusion of the softer phase from under the indenter tip ("family b" behavior). In our case, the more controlled indentation conditions and the use of spherical indenters to induce phase transformations in a-Ge resulted in a clear delineation between "family a" and "family b" behavior. In contrast, the extreme indentation conditions involved in the previous dc-Ge studies appear to result (in many cases) in both "family a" and "family b" behavior occurring during a single indent, thus complicating interpretation of the data. Indeed, the studies of Jang et al. support this proposal since, from Raman mapping, they found crystalline Raman peaks corresponding to high pressure Ge phases in the center of the indented zone. ${ }^{22,32}$ They tentatively interpreted one of the phases as bc8-Ge although they noted that the Raman peaks were not well assigned in the literature. They showed that this phase was unstable and appeared to transform to heavily strained dc-Ge since the residual Raman peak was significantly shifted to lower wave number compared to the starting dc-Ge peak at $301 \mathrm{~cm}^{-1}$. Our recent work, where we compare the Raman signatures of the various Ge phases (and XTEM data) with density functional perturbation theory calculations, ${ }^{25,26}$ shows that the initial unstable Ge phase is not bc 8 but $\mathrm{r} 8$ and the stable end phase is hd-Ge and not strained dc-Ge. Gogotsi and coworkers ${ }^{12,21,23}$ also occasionally observed similar Raman signatures to those obtained by Jang et al. using sharp indenters under high load conditions and noted that it was difficult to induce transformations to high pressure phases in dc-Ge. They suggested a range of possibilities for such phase identification, such as st12 and bc8, but also left open the possibility of unstable r8-Ge and a stable hd-Ge end phase. ${ }^{21}$ Examination of their Raman signatures would suggest that unstable $\mathrm{r} 8-\mathrm{Ge}$ and stable hd-Ge were indeed the most likely phases under the indenter tip, along with the previously mentioned st12 phase that could 
arise from fast unloading in cases where extrusion occurred ("family b" behavior) under high shear conditions. In terms of previous indentation work in a-Ge by Patriarche et al. ${ }^{14}$ and Oliver et al. ${ }^{15}$ both studies clearly observed phase transformations, but the authors did not appreciate the possibility of different transformation pathways. The TEM data in the former study, which used pointed Berkovich and Vickers indenters, showed both dc-Ge and st12-Ge end phases, with the latter observed under the highest load conditions. However, careful analysis of the Raman peak assignments from this study suggests that there is almost certainly hd-Ge and bc8-Ge present. In the case of Oliver et al., ${ }^{15}$ the TEM data again clearly showed a dc-Ge end phase for loading to maximum loads just above the pop-in load with a $4 \mu \mathrm{m}$ radius spherical indenter. It would seem clear that the data from both of these previous studies indicate "family b" behavior under conditions that favor extrusion of $(\beta-\mathrm{Sn})-\mathrm{Ge}$ and transformation to either dc-Ge or st12-Ge, depending on the maximum load and indenter shape used. However, under the indentation conditions used in both of these studies it might be expected that "family a" behavior would also be probable in the confined region under the indenter tip, as in the current study. Indeed, the possible misinterpretation of Raman assignments in the Patriache et al. case and careful examination of the loading curves and the Raman spectra in the Oliver et al. study ${ }^{15}$ strongly suggests some "family a" behavior similar to the current paper. Indeed, in the Oliver et al. case, a similar slope of the loading curve before and after pop-in and a Raman peak that was broad and shifted to the low wave number side of the $301 \mathrm{~cm}^{-1}$ dc-Ge peak, is evidence for "family a" behavior, with the latter observation strongly suggesting a hd-Ge end phase. Thus, these two previous a-Ge studies appear to be entirely consistent with the current work. Overall, taking account of the fact that most previous indentation studies of dc-Ge used extreme indentation conditions that favored both transformation pathways in Fig. 9, that there was no previous understanding that different transformation pathways may occur depending on the indentation conditions, and noting the common misinterpretation of Raman signatures for the $\mathrm{r} 8$ and hd-Ge phases as bc8 and dc-Ge, respectively, there is now reasonable consistency between previous works and the results of the current study.

Based on the comparison of our "family b" data (trace st12-Ge) with previous indentation studies under fast depressurization at high shear, we have included the possibility of a st12-Ge end phase in our transformation pathways summary (Fig. 9). In addition, whereas our indentation conditions resulted in the clear delineation between "family a" and "family b" behavior, more severe indentation conditions (i.e., high load and load rate with sharp indenter tips) are likely to exhibit both types of behavior and hence a mix of end phases will be observed depending on the precise unloading conditions. Although our proposed transformation pathways for the "family a" and "family b" cases are consistent with our data and those of others, to confirm the proposed phase evolution in each case, in-situ measurements are needed that probe the structure of the transformed phases under pressure. We have planned in-situ electrical measurements to try to establish the conductivity differences between the two families after pop-in and on unloading. In addition, in-situ Raman measurements could help to determine the phase evolution under indentation pressure, similar to recent measurements for $\mathrm{Si}^{33}$

\section{CONCLUSIONS}

We have shown that when ion implanted a-Ge films on dc-Ge substrates are subjected to indentation with a spherical tip, despite significant plastic deformation, it is possible to pressure-induce phase transformations under the indenter. This event is sudden, it involves the phase transformation of a large volume of a-Ge, and it is signified by a substantial pop-in in the loading curve. Two groups of behavior are observed during indentation. In one case (family a), the volume of metallic $(\beta$-Sn)-Ge phase that forms at pop-in is totally confined under the indenter tip. During unloading this metallic phase progressively transforms to the r8-Ge phase. However, the $\mathrm{r} 8$ phase is unstable at room temperature and pressure and further transforms to a hd-Ge phase. In the other case (family $b$ ), the soft metallic ( $\beta$-Sn)-Ge phase is not confined under the indenter at pop-in and is extruded out from under the indenter tip. In this case, the extruded $(\beta$-Sn)-Ge phase can trigger a direct transformation to dc-Ge. We suggest that this latter behavior may be related to the previously observed explosive crystallization of a-Ge under mechanical impact. Such an "explosive" crystallization process can drive (or nucleate) full transformation of remaining metallic ( $\beta$-Sn)-Ge to dc-Ge on unloading.

\section{ACKNOWLEDGMENTS}

We thank the Australian Research Council for funding. J.E.B. further acknowledges the ARC for a Future Fellowship. We also acknowledge the support of the Australian National Fabrication Facility (ANFF), ACT Node in carrying out this research.

${ }^{1}$ M. Imai, T. Mitamura, K. Yaoita, and K. Tsuji, High Pressure Res. 15, 167 (1996).

${ }^{2}$ O. Shimomura, S. Minomura, N. Sakai, K. Asaumi, K. Tamura, J. Fukushima, and H. Endo, Philos. Mag. 29, 547 (1974).

${ }^{3}$ A. Mujica, A. Rubio, A. Muñoz, and R. J. Needs, Rev. Mod. Phys. 75, 863 (2003).

${ }^{4}$ D. Daisenberger, T. Deschamps, B. Champagnon, M. Mezouar, R. Q. Cabrera, M. Wilson, and P. F. McMillan, J Phys Chem 115, 14246 (2011).

${ }^{5}$ B. Haberl, M. Guthrie, D. J. Sprouster, J. S. Williams, and J. E. Bradby, J. Appl. Crystallogr. 46, 758 (2013).

${ }^{6}$ R. J. Nelmes, M. I. McMahon, N. G. Wright, D. R. Allan, and J. S. Loveday, Phys. Rev. B 48, 9883 (1993).

${ }^{7}$ M. Hanfland and K. Syassen, High Pressure Res. 3, 242 (1990).

${ }^{8}$ F. Coppari, J. Chervin, A. Congeduti, M. Lazzeri, A. Polian, E. Principi, and A. Di Cicco, Phys. Rev. B 80, 115213 (2009).

${ }^{9}$ D. R. Clarke, M. C. Kroll, P. D. Kirchner, R. F. Cook, and B. J. Hockey, Phys. Rev. Lett. 60, 2156 (1988).

${ }^{10}$ J. E. Bradby, J. S. Williams, J. Wong-Leung, M. V. Swain, and P. Munroe, J. Mater. Res. 16, 1500 (2001).

${ }^{11}$ B. Haberl, J. E. Bradby, M. V. Swain, J. S. Williams, and P. Munroe, J. Appl. Phys. 100, 013520 (2006).

${ }^{12}$ A. Kailer, K. G. Nickel, and Y. J. Gogotsi, J. Raman Spectrosc. 30, 939 (1999).

${ }^{13}$ J. E. Bradby, J. S. Williams, J. Wong-Leung, M. V. Swain, and P. Munroe, Appl. Phys. Lett. 80, 2651 (2002). 
${ }^{14}$ G. Patriarche, E. Le Bourhis, M. M. O. Khayyat, and M. M. Chaudhri, J. Appl. Phys. 96, 1464 (2004).

${ }^{15}$ D. J. Oliver, J. E. Bradby, S. Ruffell, J. S. Williams, and P. Munroe, J. Appl. Phys. 106, 093509 (2009).

${ }^{16}$ E. Principi, F. Decremps, A. Di Cicco, F. Datchi, S. De Panfilis, A. Filipponi and A. Polian, Phys. Scr. T115, 381 (2005).

${ }^{17}$ A. Di Cicco, A. Congeduti, F. Coppari, J. C. Chervin, F. Baudelet, and A. Polian, Phys. Rev. B 78, 033309 (2008).

${ }^{18}$ O. I. Barkalov, V. G. Tissen, P. F. McMillan, M. Wilson, A. Sella, and M. V. Nefedova1, Phys. Rev. B 82, 020507(R) (2010).

${ }^{19}$ S. Minomura, J. Phys. Coll. 42, C4-181 (1981).

${ }^{20}$ G. M. Pharr, W. C. Oliver, R. F. Cook, P. D. Kirchner, M. C. Kroll, T. R. Dinger, and D. R. Clarke, J. Mater. Res. 7, 961 (1992).

${ }^{21}$ A. Kailer, Lokale Schädigung von Oberflächen und Phasenumwandlungen in harten, spröden Materialen verursacht durch mechanischen Kontakt (Shaker Verlag, Aachen, 1999).

${ }^{22}$ J. Jang, M. J. Lance, S. Wen, and G. M. Pharr, Appl Phys. Lett. 86, 131907 (2005).

${ }^{23}$ Y. Gogotsi, V. Domnich, S. N. Dub, A. Kailer, and K. G. Nickel, J. Mater. Res. 15, 871 (2000).
${ }^{24}$ D. J. Oliver, J. E. Bradby, J. S. Williams, M. V. Swain, and P. Munroe, Nanotechnology 19, 475709 (2008).

${ }^{25}$ B. C. Johnson, B. Haberl, S. Deshmukh, B. D. Malone, M. L. Cohen, J. C. Callum, J. S. Williams, and J. E. Bradby, Phys Rev. Lett. 110, 085502 (2013).

${ }^{26}$ J. S. Williams, B. Haberl, S. Deshmukh, B. C. Johnson, B. D. Malone, M. L. Cohen and J. E. Bradby, Phys. Status Solidi (RRL) 7, 355 (2013).

${ }^{27}$ S. Roorda, C. Martin, M. Droui, M. Chicoine, A. Kazimirov, and S. Kycia, Phys. Rev. Lett. 108, 255501 (2012).

${ }^{28}$ T. Takamori, R. Messier, and R. Roy, Appl. Phys. Lett. 20, 201 (1972).

${ }^{29}$ H. Leamy, W. L. Brown, G. K. Celler, G. Foti, G. H. Gilmer, and J. C. C. Fan, Appl. Phys. Lett. 38, 137 (1981).

${ }^{30}$ D. J. Oliver, J. E. Bradby, J. S. Williams, M. V. Swain, and P. Munroe, J. Appl. Phys. 105, 126101 (2009).

${ }^{31}$ B. Haberl, M. Guthrie, B. D. Malone, J. S. Smith, S. V. Sinogeikin, M. L. Cohen, J. S. Williams, G. Shen, and J. E. Bradby, Phys. Rev. B (to be published).

${ }^{32}$ J. Jang, M. J. Lance, S. Wen, J. J. Huening, R. J. Nemanich, and G. M. Pharr, Mater. Res. Soc. Symp. Proc. 841, R10.9.1 (2005).

${ }^{33}$ Y. Gerbig, C. Michaels, A. Forster, and R. Cook, Phys Rev B 85, 104102 (2012). 